\title{
TOWARDS RESPONSIBLE DESIGN WITH INTERNET OF THINGS DATA
}

\author{
Bourgeois, Jacky; Kortuem, Gerd
}

TU Delft

\begin{abstract}
Recent advances in sensing and networking technologies, namely the Internet of Things (IoT), have become key enablers of data-intensive design processes. However, the recent introduction of the General Data Protection Regulation (GDPR) in Europe has raised concerns that the GDPR might hamper dataintensive design processes. In this paper, we map the challenges of enabling ethical and compliant design of product-service systems with personal IoT data. Specifically, we present a 4-year project led by EON, an international energy provider, to design innovative home energy systems that leverage emerging technologies such as solar panels, electric vehicles and home batteries. We present our 6-stage approach to design, centred on IoT data. We highlight the barriers of responsible design with data and identify three novel trust principles for compliant use of personal IoT data in design (private-by-default, analytics transparency and Accountable analytics).
\end{abstract}

Keywords: Data-Centric Design, Design process, Ethics, Sustainability, Design

\section{Contact:}

Bourgeois, Jacky

TU Delft

Industrial Design Engineering

The Netherlands

J.Bourgeois@tudelft.nl 


\section{INTRODUCTION}

Recent advances in networked technologies (the Internet of Things) and data science methods have opened up unprecedented opportunities for modelling and analysing human behaviours (Barlacchi et al., 2017, Lazer et al., 2014). Techniques like reality mining (Eagle and Pentland, 2006) are now widely used in science to analyse data streams from mobile phones and connected sensors with the goal to identify behavioural patterns and daily routines. Examples include research to understand daily behaviours of dementia patients (meal preparation, hygiene and moving around the house) (Bradford and Zhang, 2016, Thorpe et al., 2017), and daily transportation and mobility behaviours (Calabrese et al., 2014, Zhang et al., 2013). Connected to each individual, personal IoT data offers a valuable tool to inform and evaluate. It changes the way of conducting research, increasingly relying on data as a starting point to help identify relevant needs and challenges (Apple Inc., 2016, Bourgeois et al., 2014, Handte et al., 2016). From the design perspective, it is a promising avenue complementing the set of available resources in the designers toolbox towards the design of relevant products and services. Companies already use data from connected devices and services to understand product use and optimise product design (van Kollenburg et al., 2017). Such data provides unprecedented insights into the behaviour and social interactions of people. There is an increasing interest from the design research community to understand how product and interaction designers can engage with sensor data and how sensor data can be incorporated in design processes, i.e. how data can be used as creative 'design material' (Dove and Jones, 2014, Speed and Oberlander, 2016).

However, the collection, analysis and interpretation of personal IoT data can have significant impact on the data subject's privacy. Inspired by medical science, most technical universities have recently implemented Human Research Ethics Committees (HREC) (Koepsell et al., 2015) in an attempt to ensure the protection of participants interests, safety and privacy. The Global Data Privacy Regulation (GDPR) (European Data Protection, 2016), activated in May 2018, added a stronger legal dimension into this landscape. The GDPR requires any organisation handling individual data to make this data accessible to and removable by the concerned individual as well as being transparent on its use and purpose. While the GDPR is broadly welcomed, it has raised concerns that it might hamper data-intensive design (Grossi et al., 2018, Rumbold and Pierscionek, 2017) (Mostert et al., 2016, p. 956).

Aiming for a wide use of data in design to foster the development of relevant products and services, our research focuses on identifying and addressing the challenges that prevent this approach. What are the barrier to responsible design with data? In this paper, we reflect on a case study in which data was at the centre of the design process, taking part of every stage. The 'Thinking Energy' project was a 4-year long initiative led by EON, an international energy provider. The goal was to design new home energy systems that leverage emerging technologies such as solar photovoltaic, electric vehicles and home batteries. We present the our 6-stage iterative design process and the opportunities of our approach centred on personal IoT data. Then, we map the barriers preventing responsible design with data by highlighting our irresponsibilities. Finally, we propose three trust principles to ensure a responsible use of data in research and design, pointing at a research gap.

\section{LITERATURE REVIEW}

\subsection{Data and ethics}

The use of personal IoT data in research and design brings ethical concerns. In a medical context, Vayena and Tasioulas (2016) point out that the challenge of 'ethics of big data' privacy is reduced to the right to privacy. They continue with the right to science: the ability to contribute to extending knowledge. They stress the need for tools that enable dynamic ethics supporting both right through information and fine-tuned sharing options. Both European (ALLEA, 2017) and National Codes of Conduct for Research Integrity provide guidance on principles such as honesty, scrupulousness, transparency, independence and responsibility (Algra et al., 2018, draft translation). Thus, researchers have in hand both to follow this guidance while delivering grand breaking research.

As a support to researchers, and inspired by the medical research, many institutes conducting technical research have recently implemented Human Research Ethics Committees (HREC) (Koepsell et al., 2015). The HREC is in charge to review research proposals in order to protect the participants interests, safety and privacy. Munteanu et al. (2015) point out the disconnection between between the protocols 
approved by HREC and the reality. In addition, Tolmie et al. (2016) highlight that single-time consent is not appropriate for personal IoT data as data subjects discover and understand the value and the risk of sharing their data as the study goes on. While ethical and compliance concerns are rising, the needs and challenges of technical science research with personal IoT remained unclear.

\subsection{Privacy-preserving strategies}

Considered as a grand challenge of the IoT, privacy-preserving mechanism is an active topic of research. On the one hand, watermarking techniques introduced for the media industry are ways to embed access rights directly into the data which is explored as a solution to keep access control over personal IoT data (Zhang et al., 2017). On the other hand, full encryption techniques are also researched to enable data processing without accessing the plain data (Chen et al., 2018, Sharma et al., 2018). While these different technologies could open new routes for technical scientific research with personal IoT data, it requires a deeper investigation of the designers' and researchers' needs in to better understand the practical challenges and formulate potential directions.

Looking from a Ubiquitous Computing and HCI perspective, the approach focus on the control over personal IoT data - the choice of sharing. Encouraging and supporting a right to privacy that is compatible with the right to science requires first to ensure data subjects are in control of their data. A promising direction is the data box. Perera et al. (2016) and Crabtree et al. (2017) have defined the IoT databox model as a principal means of enabling accountability and providing individuals with the mechanisms needed to build trust in the IoT. While the databox model enables a better control over personal IoT data, the data is still shared with the data requester, leaving the data subjects with the trust that their personal IoT is used only for what it was shared.

\subsection{Reproducibility}

The ability to keep track and reproduce results is a key aspect of design and research quality. It provides confidence in the outcomes and the possibility to compare results and approaches. However, studies highlights how few research results can actually be reproduced and different domains Baker and Penny (2016), Begley and Ellis (2012). Open data is especially encouraged as an enabler of reproducibility. As it remains challenging, expensive and time consuming to collect large scale IoT datasets, opening and sharing them with the scientific community is highly valuable. For example, the Extra Sensory dataset Vaizman et al. (2018) provides sensor data from smart phones and smart watches of 60 users, along user activity labelling and states. While such datasets widen opportunities for researchers, it comes as a tension with data privacy challenges.

Beyond open datasets, reproducibility is also about the research environment, the procedures and the parameters used throughout the data analysis process. Without capturing the many steps and contextual elements of this process, reproducibility cannot take place. In the field of data science, this is a challenge addressed with data-driven workflow Atkinson et al. (2017). Platforms such as myResearch enable to share these workflow with the community. The use of computer science tools is also increasingly involved including version control and Stanisic et al. (2015). In contrast with the open data challenge, reproducibility aligns with the need for accountability.

\section{CASE STUDY OF IOT PRODUCT / SERVICE DESIGN}

In 2012, the field of design for home energy management changed focus. Moving away from energy monitoring, effort shifted toward emerging technologies such as local electricity generation (e.g. solar photovoltaics, wind turbines), electric vehicles and home batteries Pierce and Paulos (2012). Pierce and colleagues stressed the need to design new data products, services and systems, especially toward new ways of using energy. For instance, local generation and storage provide opportunities beyond 'using less' energy, such as shifting the time of consumption, sharing energy or combining with electric mobility.

\subsection{Overview}

EON, an international energy provider, dedicated a 4-year project so called 'Thingking Energy' to better understand this new home energy context. Their goal was to design innovative services for solar PV households. This European-wide trial begun in early 2012 with 75 participating households around 


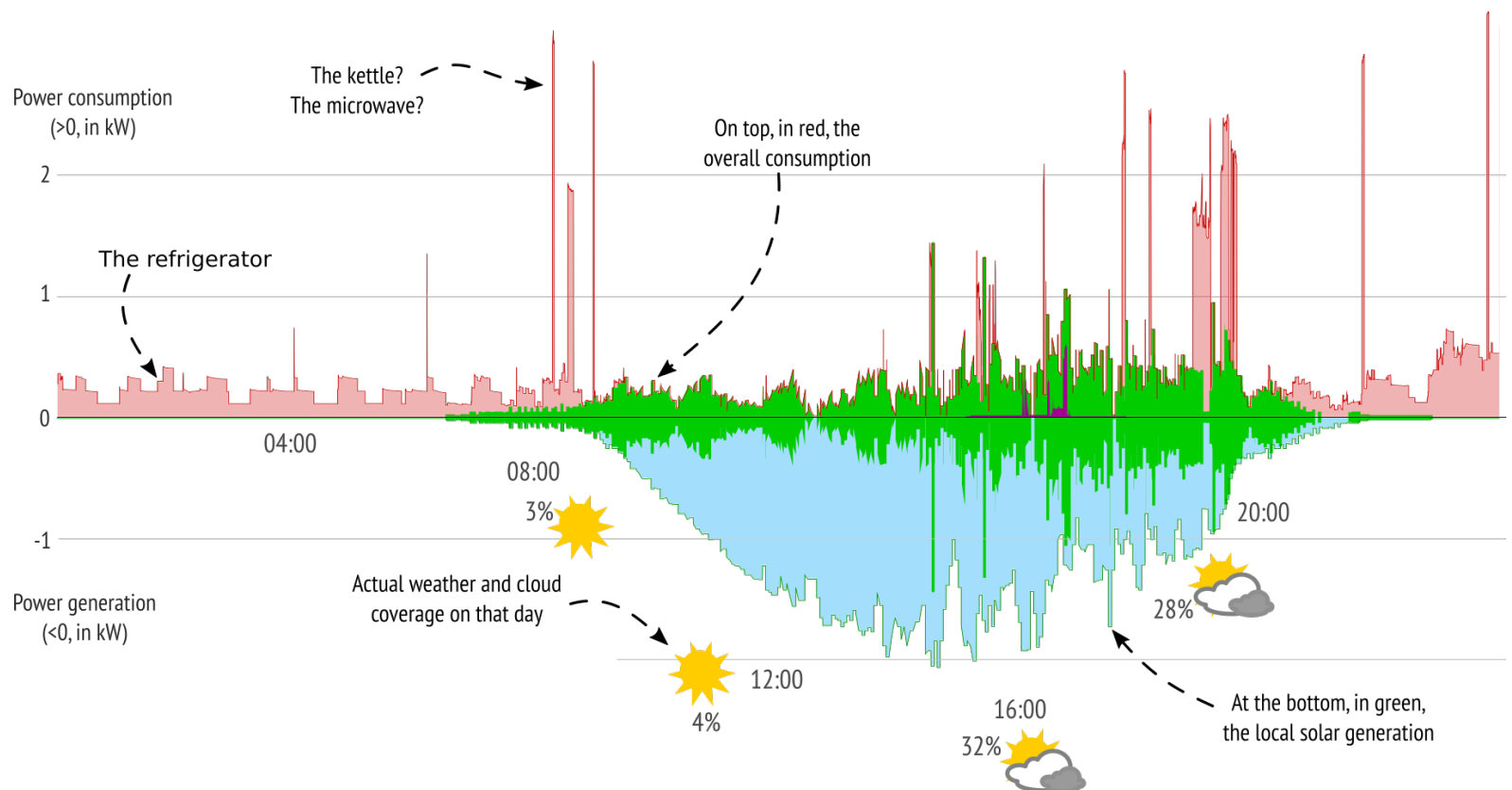

(a) Instant power in participating household 12 on July 15th, 2013. Power consumption (red, positive line), power generation (light blue, negative line) and self-consumption (dark green, mirrored line at the centred).

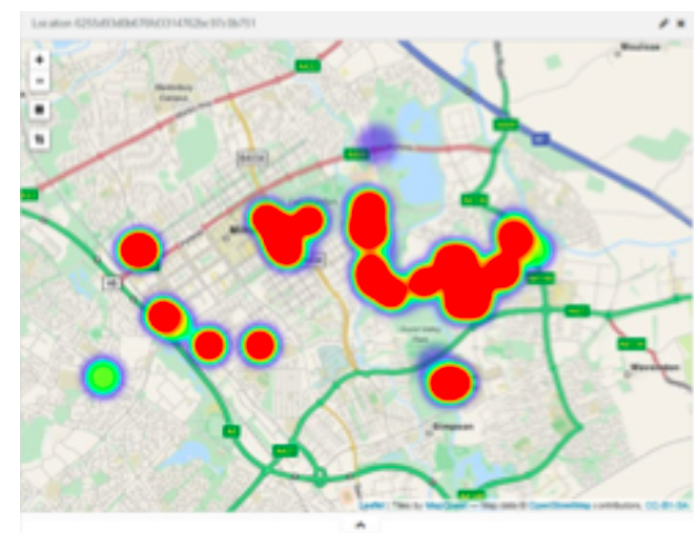

(b) Heat map of most frequent location of the Electric Vehicle of a participating household.

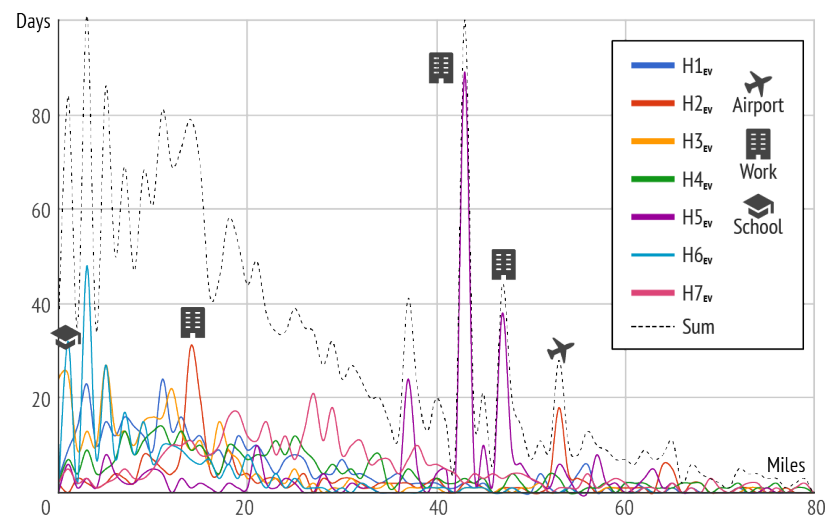

(c) Number of miles per day for seven participating households. Identified significant activities are marked with an icon, the overall doted line represent the sum of all participants

Figure 1. Examples of data collection

Milton Keynes (UK). Thinking Energy aims to understand how households can manage domestic energy use through technologies ranging from smart meters and smart plugs to smart washing machines and Electric Vehicles. The keen interest of EON in this project highlights the benefits an energy provider can get out of a better understanding of their customers.

The project represented an investment of $£ 1.5 \mathrm{~m}$ per year and approximately $3.5 \mathrm{k}$ Man-Days over 4 years. This included a dedicated team organising and coordinating, partner with Internet of Things companies for sensor and smart home network infrastructure, on the ground and online help desk support, a consulting firm organising individual interviews and workshops, collaboration with Universities for technology exploration and proof of concepts, automotive and appliance manufacturers for integration of smart products, hardware and software engineering teams with blur boundaries between regular customers and participants of the trial.

The recruitment process went as follows: Reaching out to 6000 households via email and door-todoor posting, 108 households shown interest by registering through the National Energy Foundation website. An home survey selected 75 houses suitable for the project. Participant were not necessarily EON customers, but located around Milton Keynes, a medium-sized UK suburban city. Some of the 
participating households already had solar panels installed before or installed them during the trial, and most had a keen awareness of energy issues and energy bills.

Participants signed an agreement to take part of the project. They had the ability to withdraw from the project when they wanted. Intellectual Property agreements between EON and its partners gave researchers access to participating households data. In addition, explicit consents from householders enabled partners to contact them for studies. Householders signed an explicit consents to participate in a study. This process was framed by an approval of the ethics committee consulted at the beginning and extended during the project.

\subsection{Data infrastructure}

A key characteristic of the project was its data-intensive approach, combining a wide range of sensor data collected throughout the project in combination with qualitative data. The infrastructure deployed in each participating household allowed the monitoring and the control of appliances in the house.

- Four meters were measuring the imported electricity from the grid (the typical fiscal meter), the generated electricity from the solar panels, the exported electricity to the grid and the gas consumption, recording data every three minutes;

- About ten smart plugs which monitor the individual electricity consumption of appliances. A smart plug sits between a power socket and an appliance power plug, with the ability to monitor the power consumption and to switch on and off the power;

- Temperature and humidity sensors in multiple rooms;

- A gateway was receiving energy data from the meters and smart plugs via Z-Wave and forwards this to a the EON cloud infrastructure as a typical router.

In addition, a sub group received a connected washing machine that can be monitored (programme cycle, pattern of load, etc.) and controlled (start, pause, delay, etc.) via ZigBee while another subgroup received an Electric Vehicles. Figure 1 offers a glimpse of the data collection.

\subsection{6-Stage design process}

In this project, sensor data was the core material of an iterative design process. Figure 2 illustrates this 6-stage design process centred on data.

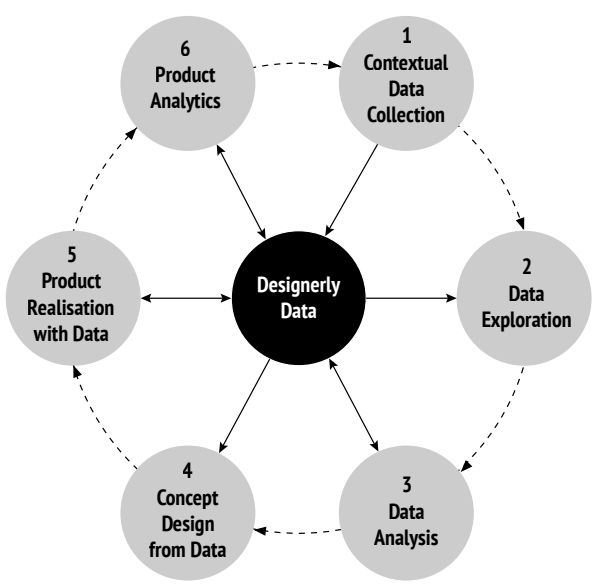

Figure 2. Iterative loT product design process with data-centric design activities

1. Contextual data collection - Each design iteration started with the collection of raw data from sensors or the gathering of data outcomes from previous iteration. This data complemented the qualitative data collected through interviews and focus groups, informal discussion during technical visits and exchange of emails.

2. Data exploration - Data exploration served multiple purpose, depending on the design iteration. It was conducted by extensive generation of charts and discussions among designers. We used it to understand or validate our understanding of the home energy system, to validate and correct the quality of sensor data, and to widely explore the qualities of data and its opportunities to drive further data analysis. 
3. Data analysis - Triggered by the outcomes of the data exploration, we built multiple forms of data analysis including aggregation, statistics, simulations of scenarios or predictions to deepen our understanding of what is happening in the households, to determine which strategy could lead to a 'best behaviour', to model trends over all householders, and to develop prediction algorithms.

4. Concept design from data - This deep understanding of energy and householders behaviour led to design concepts, blending qualitative and quantitative data. This stage is key in the transfer from 'what is happening' to 'why it is happening'.

5. Product realisation with data - Depending on the iteration, data products ranged from abstract to concrete and from simulated to actual.

6. Product Analytics - In this last stage of the design process, we deployed the data products in households to either evaluate the design or collect research insights (Research through Design). While we relied on prototypes, the process was of a Minimum Viable Products (MVP). We defined metrics along the data to record interaction and the contextual situation

\subsection{Iterative process}

A key element to designing with data is the ability to iterate on the design of a product service system in a never-ending design process. Throughout the Thinking Energy project, design iterations took many forms. The role, time and effort dedicated to each stage varied depending on the objective of each design iteration. The following examples illustrate some of these iterations.

Energy Awareness - The first design iteration aimed at generating energy awareness for householders by collecting and displaying energy consumption and generation. It started with the deployment of the smart meters and smart plugs (1). monitoring and visualising the data (2). As the data comes in, we performed deeper analysis to evaluate and validate the quality of data (3). It took a year to robustly and reliably collect data from most participants. The design and implementation of the sensing infrastructure was an integral part of the design process. In co-design session, householders and designers created initial data visualisation (4) which led to the realisation of dedicated device showing instant and past electricity consumption and generation (5). Deploying this dedicated device quickly emphasise the complexity of the relationship between generation and consumption (6).

Relationship between Consumption and Generation - Another design iteration focused on the relationship between electricity that is generated locally, on the roof from solar photovoltaic, and the electricity that is consumed by the householders. This local generation brings a new dimension to home energy as it is no longer about reducing consumption but balancing this consumption with the local generation. This balance influence the net financial cost for the household, the environmental impact and the energy efficiency (1). The data exploration helped us to characterise and understand this complex dynamic (2). Then we focused on the washing machine, an appliance available in all participating households. For each household, we used the overall consumption and generation in combination with the washing machine electricity consumption to identify what would have been the best time to run each washing machine load (3). We combined the outcome with contextual information such as the weather, the sun set and sun rise into monthly visualisation for each householders (4). We used these visualisations of what happened (i.e washing machine time) to interview householders and deepen our understanding of why it happened (5). Finally, we evaluated the potential shifting from the actual washing machine time to the best (6). This design iteration provided clear insights on the obstacle towards energy demand-shifting.

Energy Demand-Shifting - Towards the end of the project, our iterations focused on higher level information and the development of tools to support energy demand-shifting - shifting the use of electrical devices to times when solar electricity generation is at its highest. Relying on a connected washing machine, we collected specific washing programme and its properties (1). We characterised washing machine events in relation to the solar electricity generation (2). It helped us to develop prediction and recommendation algorithms (3). We designed intervention strategies as reactive (e.g. information after a washing machine event), proactive (e.g. recommendation for future washing machine use) and inuse (e.g. automation of the washing machine load) (4). Finally, We implemented and deployed these data-driven strategies via text messages and dedicated devices (5) and we monitored their use and impact (6). 


\section{DESIGNERLY DATA IRRESPONSIBILITY}

While the 'Thinking Energy' project took place before the application of the GDPR, major elements of the design process would not be legal anymore under the GDPR. They would have significantly diminished the outcomes of the project. However, we believe that the project has been conducted with integrity and the best intentions towards participating householders. As a company delivering services to customers, EON complied with legal and security aspects of the project. As a common academic research practice, we followed well-prescribed ethics procedure in order to gain permission to collect and use personally identifiable information (PII).

In this section we highlight the irresponsibilities of our design process, mapping the challenges to be addressed in order to responsibly design with personal IoT data.

- Oversight - The company's legal department as well as the research Ethics Committee of the university had limited oversight of IoT data collection and processing taking place throughout the project. Data was collected and generated through many different channels. With the GDPR, both corporate and academic Data Protection Officers (DPO) need more visibility on the collection and processing of data in order to guaranty the organisation compliance and the protection of participants / customers interests.

- $\quad$ Ethics - The company's legal department as well as research Ethics Committee of the university had limited understanding of privacy implications of IoT data analysis performed by designers and researchers, especially with respect to the potential for mashing up and analysing data from different sources. Understanding the potential ethical issues and addressing them is an important part of the design process.

- Insights - Participating householders had limited insight into personally identifiable information collected and generated about them. Although the project generated a vast amount of visualisation and feedback tools, participants had no concrete opportunity to get an overview and trace of what is collected, what is processed, what is discovered or what is done with the results.

- Consent - Data subjects were unable to provide informed consent to data collection from either University or industrial partners as they lacked an understanding of privacy implications. The consent forms signed during the project included on the infrastructure being deployed, type of data collected, the interventions to be conducted. However, there was a major pitfall: most of the design iterations were exploratory, implying unknown about the data, the processing and the potential outcomes. Beyond data subjects, designers as part of a design process might not know themselves what can be discovered as it is the goal of the exploration.

- $\quad$ Access - the 'Thinking Energy' project leveraged EON infrastructure. Participating households were part of the EON system as regular customers. This is a viable route to conduct such a large scale project. As part of a never-ending design process at scale, participants were accessing regular. However, customer systems are not designed to be accessible. There was no compartment between data with and without consent, thus no easy compartment between participants of the projects and the rest of the (many) EON customers. To access data from these participants, EON had to develop a tool enabling designers to extract data of the given participants from their corporate database. It was also challenging to collect new type of data as part of the product analytics (stage 6).

- Sharing - Sharing of IoT data between partners and beyond was difficult as each data set came with unique ethics and consent conditions, mainly as part of bilateral agreement. With the GDPR, none of the collected data can be reused under the current consent.

\section{DISCUSSION}

Our list of data irresponsibility does not aim towards a critic against the GDPR nor the use of personal IoT data in research and design. We see the GDPR as a step forward and an opportunity to develop design processes that leverage opportunities of data in design while complying with the GDPR.

\subsection{Trust principles}

It stresses the need for a different approach to designing with data to overcome these data irresponsibility. To this end, we formulate three key trust principles for such approach:

1. Private by default: researchers and designers cannot use personally identifiable data unless data subjects have given voluntary, explicit and informed consent; 
2. Analytics transparency: researchers and designers must disclose their analytics algorithms, making them reviewable and traceable by relevant stakeholders;

3. Accountable analytics: data analytics must be performed in a trusted environment that guarantees its analytics processes and the control over the personal IoT data.

Principle 1 states that data subjects should have the opportunity to provide or refuse consent to the use of data by researchers and designers before it can be accessed by researchers. This principle is especially relevant for opportunistic data which is initially collected for purposes other than research and design. In our case study smart meters, enabling energy providers to effectively manage the grid, also generate opportunistic data for researchers and designers.

Principle 2 states that analytics algorithms should be open for scrutiny in a conversational environment that encourages multiple perspectives. This is relevant since privacy aspects can only be understood if it is transparent, which data is analysed and how, especially when mashing up data from multiple sources. Principle 3 states that analytics algorithms and data requirements should be combined and executed autonomously in a sealed environment. This is relevant to ensure that the reviewed algorithm is the one executed and to prevent data leaks.

\subsection{Research gap}

The development of research and design methods that align with these three trust principles is underexplored and requires more attention. This is a research gap at the intersection of design and research methods, data management, and data control. It aligns with three key stakeholders: (i) researchers and designers, (ii) organisations including ethics committees, legal departments and ICT departments, (iii) and data subjects.

As briefly highlighted in the literature review, there is existing research on data-enabled design (Dove and Jones, 2014, Speed and Oberlander, 2016, van Kollenburg et al., 2017), privacy and inform consent (Crabtree et al., 2017, Munteanu et al., 2015, Perera et al., 2016, Tolmie et al., 2016, Vayena and Tasioulas, 2016) as well as reproducibility and accountability (Atkinson et al., 2017, Stanisic et al., 2015). However, key research questions lie at the intersection of those domains which cannot be addressed from a single perspective. For instance, What are the core values of each stakeholder and their responsibility regarding the use of data in research and design? How can we mitigate and balance the value-conflicts underlying these responsibilities? How do we effectively operationalise the responsibilities of all stakeholders?

\section{CONCLUSION}

In this paper we illustrated the implementation of an iterative design process centred on IoT data with a large-scale case study on home emerging energy technologies. We highlighted the role of personal IoT data as a critical design material. We contrasted the benefits and opportunities of our approach with a reflection on our data irresponsibilities, pointing at its legal, ethical and organisational clashes and flaws. Finally, we identified a research gap to be explored in order to address three trust principles: privacy by default, analytics transparency and accountable analytics.

\section{REFERENCES}

Keimpe Algra, Lex Bouter, Antoine Hol, and Jan van Kreveld. Netherlands Code of Conduct for Research Integrity for consultation purposes. Technical report, 2018. https://www.vsnu.nl/en_GB/netherlandscode-of-conduct-for-research-integrity.

ALLEA. The European Code of Conduct for Research Integrity. Technical report, 2017. https://ec.europa.eu/ research/participants/data/ref/h2020/other/hi/h2020-ethics_code-of-conduct_en.pdf.

Apple Inc. ReserachKit and CareKit, 2016. http://www.apple.com/researchkit/.

Malcolm Atkinson, Sandra Gesing, Johan Montagnat, and Ian Taylor. "Scientific workflows: Past, present and future”. Future Generation Computer Systems, Vol. 75, pp. 216-227, 2017. ISSN 0167739X. http://dx.doi.org/10.1016/j.future.2017.05.041.

Monya Baker and Dan Penny. "Is there a reproducibility crisis?" Nature, Vol. 533 No.7604, pp. 452-454, 2016. ISSN 14764687. http://doi.org/10.1038/533452A.

Gianni Barlacchi, Christos Perentis, Abhinav Mehrotra, Mirco Musolesi, and Bruno Lepri. “Are you getting sick? Predicting influenza-like symptoms using human mobility behaviors". EPJ Data Science, Vol. 6 
No. 1, p. 27, 12 2017. ISSN 2193-1127. http://doi.org/10.1140/epjds/s13688-017-0124-6. http://epjdatascience.springeropen.com/articles/10.1140/epjds/s13688-017-0124-6.

Glenn Begley, C. and Lee M. Ellis. "Drug development: Raise standards for preclinical cancer research". Nature, Vol. 483 No. 7391, pp. 531-533, 2012. ISSN 00280836. http://doi.org/10.1038/483531a.

Jacky Bourgeois, Janet van der Linden, Gerd Kortuem, Blaine A Price, and Christopher Rimmer. Conversations with my washing machine. In Proceedings of the 2014 ACM International Joint Conference on Pervasive and Ubiquitous Computing - UbiComp '14 Adjunct, UbiComp '14, pp 459-470, New York, NY, USA, 2014. ACM. ISBN 9781450329682. http://doi.org/10.1145/2632048.2632106. http://dl.acm.org/citation. cfm?doid=2632048.2632106.

DanaKai Bradford and Qing Zhang. How to Save a Life: Could Real-Time Sensor Data Have Saved Mrs Elle? In Proceedings of the 2016 CHI Conference Extended Abstracts on Human Factors in Computing Systems CHI EA '16, pp. 910-920, New York, New York, USA, 2016. ACM Press. ISBN 9781450340823. http://doi.org/10.1145/2851581.2851598. http://dl.acm.org/citation.cfm?doid=2851581.2851598.

Francesco Calabrese, Laura Ferrari, and Vincent D. Blondel. "Urban Sensing Using Mobile Phone Network Data: A Survey of Research". ACM Computing Surveys, Vol. 47 No. 2, pp. 1-20, 11 2014. ISSN 03600300. http://doi.org/10.1145/2655691. http://dl.acm.org/citation.cfm?doid=2658850.2655691.

Chen, F., Jiang, X., Wang, S., Schilling, L., Meeker, D., Ong, T., Matheny, M., Doctor, J., Ohno-Machado, L. and Vaidya, J. "Perfectly Secure and Efficient Two-party Electronic Health Record Linkage". IEEE Internet Computing, (April) pp. 32-41, 2018. ISSN 10897801. http://doi.org/10.1109/MIC.2018.112102542.

Andy Crabtree, Tom Lodge, James Colley, Chris Greenghalgh, and Richard Mortier. Accountable Internet of Things? Outline of the IoT databox model. In 18th IEEE International Symposium on A World of Wireless, Mobile and Multimedia Networks, WoWMoM 2017 - Conference, pp. 1-6, 6 2017. ISBN 9781538627228. http://doi.org/10.1109/WoWMoM.2017.7974335.

Graham Dove and Sara Jones. Using data to stimulate creative thinking in the design of new products and services. Proceedings of the 2014 conference on Designing interactive systems - DIS '14, pp. 443-452, 2014. 10.1145/2598510.2598564. http://dl.acm.org/citation.cfm?doid=2598510.2598564.

Nathan Eagle and Alex Pentland. "Reality mining: Sensing complex social systems". Personal and Ubiquitous Computing, Vol. 10 No. 4, pp. 255-268, 2006. ISSN 16174909. http://doi.org/10.1007/s00779-005-0046-3.

European Data Protection. General Data Protection Regulation, 2016. https://gdpr-info.eu/.

Valerio Grossi, Beatrice Rapisarda, Fosca Giannotti, and Dino Pedreschi. Data science at SoBigData: the European research infrastructure for social mining and big data analytics. International Journal of Data Science and Analytics, 5 2018. ISSN 2364-415X. http://doi.org/10.1007/s41060-018-0126-X. http://link.springer.com/10.1007/s41060-018-0126-x.

Marcus Handte, Stefan Foell, Stephan Wagner, Gerd Kortuem, and Pedro Jose Marron. “An Internet-of-Things Enabled Connected Navigation System for Urban Bus Riders". IEEE Internet of Things Journal, Vol. 3 No. 5, pp. 735-744, 10 2016. ISSN 23274662. http://doi.org/10.1109/JIOT.2016.2554146.

David Koepsell, Willem-Paul Brinkman, and Sylvia Pont. "Human Participants in Engineering Research: Notes from a Fledgling Ethics Committee". Science and Engineering Ethics, Vol. 21 No. 4, pp. 1033-1048, 8 2015. ISSN 1353-3452. http://doi.org/10.1007/s11948-014-9568-2. http://link.springer.com/10.1007/s11948-014-9568-2.

David Lazer, Alex Pentland, Lada Adamic, Sinan Aral, Albert-László Barabási, Devon Brewer, Nicholas Christakis, Noshir Contractor, James Fowler, Myron Gutmann, Tony Jebara, Gary King, Michael Macy, Deb Roy, and Marshall Van Alstyne. "Computational social science". Science, Vol. 323, (February) pp. 721-723, 2014. ISSN 0036-8075, 1095-9203. http://doi.org/10.1126/science.1169410. http://science.sciencemag.org/content/sci/323/5915/721.full.pdf.

Menno Mostert, Annelien L. Bredenoord, Monique C.I.H. Biesaart, and Johannes J.M. Van Delden. "Big Data in medical research and EU data protection law: Challenges to the consent or anonymise approach". European Journal of Human Genetics, Vol. 24 No. 7, pp. 956-960, 7 2016. ISSN 14765438. http://doi.org/10.1038/ejhg.2015.239. http://europepmc.org/articles/PMC5070890.

Cosmin Munteanu, Heather Molyneaux, Wendy Moncur, Mario Romero, Susan O’Donnell, and John Vines. Situational Ethics: Re-thinking Approaches to Formal Ethics Requirements for Human-Computer Interaction. Proceedings of the 33rd Annual ACM Conference on Human Factors in Computing Systems, pp. 105-114, 2015. http://doi.org/10.1145/2702123.2702481. http://doi.acm.org/10.1145/2702123. 2702481.

Charith Perera, Ciaran McCormick, Arosha K. Bandara, Blaine A. Price, and Bashar Nuseibeh. Privacy-byDesign Framework for Assessing Internet of Things Applications and Platforms. Proceedings of the 6th International Conference on the Internet of Things - IoT'16, pp. 83-92, 2016. http://doi.org/10.1145/ 2991561.2991566. http://dl.acm.org/citation.cfm?doid=2991561.2991566. 
James Pierce and Eric Paulos. Beyond energy monitors. In Proceedings of the 2012 ACM annual conference on Human Factors in Computing Systems - CHI '12, p. 665, New York, New York, USA, 2012. ACM Press. ISBN 9781450310154. http://doi.org/10.1145/2207676.2207771. http://dl.acm.org/citation.cfm?doid= 2207676.2207771.

John Mark Michael Rumbold and Barbara Pierscionek. "The effect of the general data protection regulation on medical research". Journal of Medical Internet Research, Vol. 19 No.2, p. e47, 2 2017. ISSN 14388871. http://doi.org/10.2196/jmir.7108. http://www.ncbi.nlm.nih.gov/pubmed/28235748.

Sagar Sharma, Keke Chen, and Amit Sheth. "Toward practical privacy-preserving analytics for IoT and cloud-based healthcare systems". IEEE Internet Computing, Vol. 22 No. 2, pp. 42-51, 2018. ISSN 10897801. http://doi.org/10.1109/MIC.2018.112102519.

Chris Speed and Jon Oberlander. "Designing from, with and by Data: Introducing the ablative framework". Design Research Society (DRS), 2016.

Luka Stanisic, Arnaud Legrand, and Vincent Danjean. "An Effective Git And Org-Mode Based Workflow For Reproducible Research". ACM SIGOPS Operating Systems Review, Vol. 49 No. 1, pp. 61-70, 2015. ISSN 01635980. http://doi.org/10.1145/2723872.2723881. http://dl.acm.org/citation.cfm?doid=2723872. 2723881.

Thorpe, J R, Forchhammer, B H and Maier, A. "Sensing behaviour in healthcare design". Proceedings of the International Conference on Engineering Design, ICED, Vol. 3 No. (DS87-3), pp. 171-180, 2017. ISSN 2220-4342. https:/www.scopus.com/inward/record.uri?eid=2-s2.0-85029807945\&partnerID=40\&md5= 3e71151803ba396af1fc9354153b596b.

Peter Tolmie, Andy Crabtree, Tom Rodden, James A Colley, and Ewa A Luger. "This has to be the cats" Personal Data Legibility in Networked Sensing Systems. In Proceedings of the 19th ACM Conference on Computer-Supported Cooperative Work \& Social Computing - CSCW'16, CSCW'16, pages 490-501, New York, NY, USA, 2016. ACM. ISBN 9781450335928. http://doi.org/10.1145/2818048.2819992. http://dl.acm.org/citation.cfm?doid=2818048.2819992.

Yonatan Vaizman, Katherine Ellis, Gert Lanckriet, and Nadir Weibel. ExtraSensory App: Data Collection In-the-Wild with Rich User Interface to Self-Report Behavior. Proceedings of the 2018 CHI Conference on Human Factors in Computing Systems - CHI '18, pp. 1-12, 2018. http://doi.org/10.1145/3173574.3174128. https://dl.acm.org/citation.cfm?doid=3173574.3174128

Janne van Kollenburg, Sander Bogers, Eva Deckers, Joep Frens, and Caroline Hummels. How Design-inclusive UXR Influenced the Integration of Project Activities. In Proceedings of the 2017 CHI Conference on Human Factors in Computing Systems - CHI '17, CHI '17, pp. 1408-1418, New York, NY, USA, 2017. ACM. ISBN 9781450346559. http://doi.org/10.1145/3025453.3025541. http://dl.acm.org/citation.cfm? doid=3025453.3025541.

Effy Vayena and John Tasioulas. "The dynamics of big data and human rights: The case of scientific research". Philosophical Transactions of the Royal Society A: Mathematical, Physical and Engineering Sciences, Vol. 374 No. 2083, 2016. ISSN 1364503X. http://doi.org/10.1098/rsta.2016.0129.

Fuzheng Zhang, David Wilkie, Yu Zheng, and Xing Xie. Sensing the pulse of urban refueling behavior. In Proceedings of the 2013 ACM international joint conference on Pervasive and ubiquitous computing UbiComp '13, p. 13, New York, New York, USA, 2013. ACM Press. ISBN 9781450317702. http://doi.org/10.1145/2493432.2493448. http://dl.acm.org/citation.cfm?doid=2493432.2493448.

Guoyin Zhang, Liang Kou, Liguo Zhang, Chao Liu, Qingan Da, and Jianguo Sun. A New Digital Watermarking Method for Data Integrity Protection in the Perception Layer of IoT. 2017, 2017. 